\title{
Transformación social y migración internacional en la era neoliberal: una propuesta teórica
}

\author{
Social transformation and international migration \\ in the neoliberal era: a theoretical approach
}

Resumen. Este artículo plantea un marco teórico de alcance intermedio para estudiar la migración internacional contemporánea. A partir de la crítica polanyiana de la economía de mercado, se sugiere que muchas formas contemporáneas de migración, se deben comprender con relación a los profundos cambios sociales, políticos y económicos que han acompañado, la expansión de la economía de mercado en los niveles local, nacional y transnacional en la era neoliberal. Además de recalcar que la migración es una parte integral de estos procesos de trasformación social, la propuesta teórica hace hincapié en la importancia de investigar este fenómeno social desde una perspectiva multiescalar y multidisciplinaria. Así, el objetivo de este artículo es guiar y generar una discusión crítica sobre las maneras en que la movilidad humana contemporánea, en el escenario de relaciones cambiantes de poder e inequidad que configuran la era actual de la globalización neoliberal, interactúa con diversas instituciones y relaciones políticas, económicas y sociales a través de múltiples escalas socioespaciales.

Palabras clave: migración internacional, teoría, transformación social, neoliberalismo.

\begin{abstract}
This article offers a middle-range theoretical framework for the study of contemporary international migration. Deriving from the polanyian criticism of the market economy, we suggest that many current forms of migration should be understood in relation to the deep social, political and cultural changes which accompany the expansion of the market economy at the local, national and transnational level in the neoliberal era. While it re-examines migration as an integral part of these processes of social transformation, our theoretical approach also emphasizes the importance of researching this social phenomenon from a multi-level and multidisciplinary perspective. Thus, the aim of this article is to guide and generate a critical debate on the ways in which contemporary human mobility, against a backdrop of changing power relations and inequality that shape the current period of neoliberal globalization, interacts with various institutions and political, economic and social relations through multiple socio-spacial levels.
\end{abstract}

Keywords: international migration internacional, theory, social transformation, neoliberalism.

\footnotetext{
* Mexicana/australiana. Investigadora asociada del Alfred Deakin Institute for Citizenship and Globalisation, Deakin University, Australia. Correo-e: magdalena.ariascubas@deakin.edu.au
} 


\section{Introducción ${ }^{1}$}

En este artículo se expone el marco teórico que fue desarrollado como parte del proyecto de investigación sobre la Transformación Social y Migración Internacional (STIM, por sus siglas en inglés), llevado a cabo por académicos en la Universidad de Sídney (Australia), en colaboración con colegas en Corea del Sur, México y Turquía (Castles et al., 2011a; Castles, Ozkul y Arias, 2015). ${ }^{2}$ El proyecto analiza la manera en que la movilidad humana y los procesos más amplios de transformación social se han interconectado en el escenario de relaciones de poder e inequidad, cada vez más amplias y universales, las cuales a su vez han configurado la era actual de la globalización neoliberal. Este marco teórico continúa el trabajo de Stephen Castles (2010) y su llamado a discutir la complejidad, interconexión, variabilidad, trama y multidimensionalidad de los procesos migratorios que han ocurrido en el contexto de un rápido cambio global. Con base en la crítica de Karl Polanyi (2003) sobre la economía de mercado (publicada originalmente en 1944), se argumenta que las últimas cuatro décadas de globalización neoliberal han atestiguado un periodo de profunda reorganización social, política y económica en casi todas las partes del mundo (Stiglitz, 2003). ${ }^{3}$ El marco teórico aquí presentado sugiere que muchas formas contemporáneas de migración son mejor comprendidas con respecto a estos profundos cambios sociales, políticos y económicos que han acompañado la expansión de la economía de mercado en los niveles local, nacional y transnacional.

Con el objetivo de enmarcar la propuesta teorética, este artículo comienza discutiendo la expansión del interés y la investigación académica en torno de la migración internacional y algunas de sus debilidades. Posteriormente, se plantea un «lente de transformación social» como una teoría alternativa de alcance intermedio para analizar ciertas formas de migración en la era de la globalización neoliberal. Se destaca que percepciones y conocimiento clave sobre la migración contemporánea y la transformación social se pueden extraer al hacer operativo

\footnotetext{
${ }^{1}$ Agradecemos la asistencia y comentarios de Stephen Castles y Raúl Arias Cubas.

${ }^{2}$ Este proyecto ha sido un esfuerzo colaborativo de investigadores en la Universidad de Sídney (Magdalena Arias Cubas, Stephen Castles, Chulhyo Kim, Elsa Koleth, Cailin Maas, Derya Ozkul y Rebecca Williamson), junto con colegas en Corea del Sur (Hye-Kyung Lee y Doon-Hoon Seol), México (Raúl Delgado Wise y Rodolfo García Zamora) y Turquía (Mine Eder y Ahmet Icduygu).

${ }^{3}$ Véase Polanyi-Levitt (2014) para un resumen crítico de la obra de Polanyi.
} 
los conceptos desarrollados por Polanyi (2003) de la economía arraigada (embeddedness), las mercancías ficticias (fictitious commodities), y el doble movimiento (double movement). Asimismo, se enfatiza en la necesidad de reflexionar acerca de específicas deficiencias del marco polanyiano original, en particular es preciso reconfigurar el análisis más allá del marco «nacional» y considerar de modo crítico formas existentes de desigualdad que simplemente no pueden ser capturadas en su totalidad mediante un análisis de la expansión de la economía de mercado. Se insiste que, en respuesta a este escenario y en el marco del canon existente sobre la migración internacional, nuestra comprensión de la migración en la era neoliberal puede fortalecerse con fundamento en el conocimiento de la teoría social contemporánea. En la conclusión, recapitulamos cinco principios esenciales de este marco teorético de alcance intermedio que esperamos guíen y generen más discusión.

\section{El crecimiento y las limitaciones de los estudios de migración}

El interés intelectual en la migración se ha desarrollado rápidamente en los últimos 25 años, ello refleja cambios dramáticos en el volumen, las formas y las direcciones de la migración internacional. Jennifer Lee, Jørgen Carling y Pia Orrenius (2014) han identificado las siguientes áreas clave de cambio en las tendencias de investigación y en las tendencias de flujos migratorios recientes. Éstas incluyen: a) El establecimiento de la migración internacional como un propio subcampo de estudio a través de diversas disciplinas en las ciencias sociales. b) La creciente multiplicidad de los países de origen y el surgimiento de nuevos destinos. c) La creciente diversidad étnico-racial y socioeconómica de los migrantes. d) El auge de migrantes con un estatus irregular. e) El enfoque renovado en las conexiones transnacionales, incluidas las remesas y las diásporas. f) El desarrollo de modelos de integración bidireccional que explican cómo los migrantes cambian las sociedades receptoras (y no sólo cómo éstas cambian a los migrantes).g) Finalmente, el aumento en la atención a las perspectivas de los hijos de migrantes. A lo anterior habría que añadir el encuadre persistente de algunos migrantes en debates académicos y políticos como un problema o incluso una amenaza en contra de la identidad, el nivel de vida y la seguridad «nacional» (Castles y Delgado, 2008). 
Numerosas reseñas críticas han destacado (Arango, 2004; King, 2012; Massey et al., 1998; de Haas, 2014), que los estudios de la migración son un área fundamental de la investigación social que han logrado avances durante las últimas décadas. Dado que la migración internacional presenta desafíos primordiales a los estudiosos de las ciencias sociales y a aquellos que formulan políticas públicas, es importante desarrollar su propia teoría, metodología y contribución al conocimiento. A la par, es fundamental resaltar una serie de factores que dificultan el avance de dicho conocimiento, debilitan la comprensión de cambios sociales relacionados a la migración y reducen el valor de esta investigación como una guía para la formación de políticas (véase también Castles, 2010). Tales problemas comprenden:

1. El nacionalismo metodológico que permea las ciencias sociales (Beck, 2007) y que es un problema especialmente grave para los estudios de migración (Wimmer y Glick, 2003). En particular, la investigación acerca de la migración tiende a vincularse con experiencias históricas específicas sobre el control del movimiento de poblaciones y la diversidad en el nivel «nacional», lo que refuerza el contenedor del Estado nación.

2. La fragmentación a partir de disciplinas y paradigmas que resulta en una investigación compartimentada, con poca colaboración analítica y metodológica a través de las fronteras disciplinarias (además de los cismas que existen dentro de las mismas disciplinas). ${ }^{4}$ Adicionalmente, existe una fragmentación con base en criterios espaciales o funcionales, que resulta en divisiones entre investigaciones en torno de los determinantes de la migración e investigaciones sobre la incorporación de los migrantes (Massey et al., 1998). Esto se asocia con otras divisiones - entre enfoques en la migración interna e internacional, la migración forzada y la económica, la migración permanente y temporal, y otras - que limitan una comprensión del carácter histórico de la migración y de su arraigo en procesos de cambio social.

3. La investigación impulsada por política, en particular en países receptores del Norte, donde se ha encargado un gran volumen de investigación del tema en respuesta al crecimiento y la politización de la migración. Desde un punto de vista crítico, puede significar que las preguntas de investigación, sus métodos y sus hallazgos están conformados por intereses políticos, situación que limita su poder analítico y su impacto social (Castles, 2004).

${ }^{4}$ Véanse Brettell y Hollifield (2007), Massey et al. (1998) y Portes y DeWind (2004) como ejemplos de esfuerzos para superar esta fragmentación. 
4. El aislamiento de los estudios de migración de la teoría social contemporánea, con la exclusión de investigaciones de migración y diversidad en los debates más amplios acerca de la sociedad y viceversa. Aun recientemente, y a pesar de que la movilidad de personas en el nivel internacional es una forma crucial de la globalización neoliberal, esos tópicos recibían escasa atención en trabajos seminales (Albrow, 1996; Castells, 1996; 1997; 1998). De hecho, pocos trabajos sobre el cambio global (Bauman 2001; Beck 2007; Held et al., 1999) destacaban la centralidad de la migración en las relaciones sociales contemporáneas.

5. El dominio por epistemologías, instituciones y expertos del Norte global. Es el resultado de desigualdades persistentes de poder, lenguaje y financiamiento que conducen a la marginación de temas y metodologías relevantes en las ciencias sociales (Alatas, 2003; Connell, 2007; De Sousa, Arriscado y Meneses, 2007; Tuhiwai, 1999) y que en los estudios de migración ha limitado las cuestiones clave de interés a la seguridad, el control, la integración y el desarrollo económico (a través del impacto positivo de las remesas) (Castles y Delgado, 2008). ${ }^{5}$

\section{El lente analítico de la transformación social}

En este escenario, se propone un marco para el estudio de la migración internacional que arraigue la migración en procesos de transformación social más amplios que tienen lugar en la era neoliberal. Con ello, se reconoce que algunos aspectos de la relación entre la migración y los procesos de transformación social han sido ya parte de debates académicos. En realidad, una gran cantidad de la investigación se ha centrado en analizar el comienzo y la perpetuación de los procesos migratorios, cuestiones vinculadas directa o indirectamente a cambios más amplios en las realidades económicas, políticas y sociales de individuos, hogares, comunidades, países y regiones involucrados (Massey et al.,

\footnotetext{
${ }^{5}$ Esto no implica que académicos fuera de América del Norte o de Europa Oriental hayan sido silenciados. Se puede recalcar el trabajo de instituciones de investigación como la Unidad Académica en Estudios de Desarrollo de la Universidad Autónoma de Zacatecas (México) o el Centro de Migración Scalabrini en Manila (Filipinas). También es preciso reconocer publicaciones como Migración y Desarrollo o la Revista Interdisciplinar da Mobilidade Humana (con sede en Brasil), así como las redes de investigación, la Association of Migration Research (GAR) de Turquía o la Korean International Migration Association (KIMA) de Corea del Sur. Sin embargo, muchas de esas iniciativas son recientes y limitadas en comparación con los centros y las publicaciones académicas que examinan el impacto de la migración en los países de destino norteamericanos y europeos, los cuales influyen fuertemente en debates globales.
} 
1998). En contraste, como ya se mencionó con anterioridad, la presente investigación ha sido a menudo fragmentada con fundamento en disciplinas y paradigmas o en criterios espaciales y funcionales. Por lo tanto, defiende la necesidad de situar la investigación en torno de la migración contemporánea en una comprensión más general y multidisciplinaria de la sociedad que tome en cuenta cambios sociales, económicos y políticos que están ocurriendo a través de múltiples escalas socioespaciales. Lo anterior supone que: a) los relatos teóricos de la migración deben integrarse en una teoría social más amplia, b) la investigación de cualquier fenómeno específico de migración debe incluir un entendimiento del escenario social más general histórico y contemporáneo del fenómeno, y c) esta investigación debe vincular experiencias de migración en el nivel local con otros niveles socioespaciales (y en particular con los procesos globales) (Castles, 2010).

El punto de partida del marco conceptual es que la movilidad humana constituye un segmento normal de la vida social y que la migración es, por ende, una parte integral de los procesos de transformación social. De acuerdo con Stephen Castles (2010), la transformación social se define como un cambio en las relaciones sociales tan profundo que afecta prácticamente a todas las formas de interacción social, a todos los individuos y a las comunidades de modo simultáneo; y a la vez genera transformaciones concurrentes en el ámbito de la cultura, las relaciones e instituciones sociales, las identidades personales, la ideología, la economía y la política. En la práctica, significa invertir el proceso analítico habitual de los estudios de migración. Si bien la mayoría de las investigaciones comienza desde un enfoque en un fenómeno migratorio concreto, y luego busca sus «causas» y sus "consecuencias», el enfoque de esta investigación se sustenta en un estudio crítico de la transformación social neoliberal y muestra cómo la migración es un componente intrínseco de ese proceso, con vínculos multidireccionales y complejos a otros tipos de cambio.

Considerando esa perspectiva, es preponderante explorar los vínculos entre las dimensiones locales, nacionales y globales de la transformación social y la migración, junto con los vínculos existentes entre los fenómenos sociales, económicos y políticos. Ante todo, para teorizar sobre la migración es preciso identificar las conexiones entre las dimensiones transnacionales, nacionales y locales de la transformación social y las diversas formas de mediación que coexisten entre esas escalas socioespaciales. Así, aspectos clave de los procesos de transformación social — tales como la expansión de la economía de mercado, la elaboración 
y reelaboración de los regímenes de ciudadanía, o el desarrollo de prácticas sociales de solidaridad y resistencia - son reforzados, impugnados o son hechos significativos mediante diferentes niveles de poder y en diferentes escalas socioespaciales (Williamson, 2015). En ese sentido, y en lugar de privilegiar una escala sobre otras, es indispensable conceptualizarlas de una manera equitativa que al mismo tiempo tome en cuenta el diálogo y las tensiones entre ellas (Levitt, 2004). Es relevante observar cómo las fuerzas globales influyen en la movilidad de las personas, pero también cómo estas fuerzas son mediadas por patrones culturales o experiencias históricas en el nivel nacional y por la acción humana en el nivel local. Es notable que dicha comprensión arraigada de la migración no sólo acentúa los vínculos entre las distintas escalas, sino que crea un espacio para estudiar las «causas» y las «consecuencias» de la migración simultáneamente (en lugar de separarlas conceptualmente) (De Haas, 2014).

En adición, con la finalidad de teorizar la migración internacional se requiere identificar y asociar dimensiones trascendentales de cambio, incluyendo las dinámicas de fuerzas económicas, políticas y sociales. Al argumentar que la migración es un componente esencial del cambio social, es posible remarcar que la migración es influenciada por factores económicos, sociales y políticos complejos. Una teoría que sitúa a la economía como desintegrada de la sociedad, en la que las consecuencias políticas y sociales de las actividades económicas se tratan como «externalidades» inevitables, conduce a un conocimiento débil y desincorporado de la migración (Castles, 2015). Por ejemplo, una comprensión económica de la migración reduce a los migrantes a no más que factores de producción (Rosewarne, 2010) y en consecuencia oculta información relevante de las condiciones políticas bajo las cuales se producen ciertos movimientos humanos y del estatus social de los derechos y las libertades de los migrantes. En cambio, una comprensión arraigada de la migración, la conceptualiza como una parte integral de procesos sociales y políticos dinámicos donde las relaciones e instituciones sociales y políticas (históricas y contemporáneas) poseen la misma importancia que las económicas.

Este entendimiento integrado de la migración crea un espacio conceptual que hace hincapié en los vínculos y las tensiones existentes entre diferentes dimensiones y escalas sociales, económicas y políticas. Aparte de su relevancia conceptual, esta comprensión integrada también es de importancia política. En contraposición con la percepción dominante de la migración como un problema o una amenaza, la comprensión integrada acentúa que la movilidad humana 
—desde los flujos locales hasta los movimientos transcontinentales- ha sido un elemento integral de la vida y del cambio social a lo largo de la historia y lo continúa siendo en la era neoliberal. En palabras de Ronald Skeldon (1997), cuando se acepta que la migración es una parte integral del comportamiento de todas las sociedades en cualquier momento (y no es anormal o excepcional), el objetivo del análisis se convierte entonces en percibir cómo su forma y función se relacionan con otros cambios en las economías y sociedades. En definitiva, las migraciones no son fenómenos aislados ni anormales. Por el contrario, numerosos movimientos de poblaciones contemporáneos están estrechamente vinculados con flujos históricos y actuales de capital, de materias primas y de ideas, en formas que son explicativas de relaciones de poder e inequidad que conforman la era neoliberal.

\section{La migración internacional en la era neoliberal}

La propuesta teorética para estudiar los procesos de transformación social y la movilidad humana en la era neoliberal se basa en la tesis de Karl Polanyi (2003) de «la gran transformación» y en recientes exposiciones de su trabajo (Burawoy, 2015; Fraser, 2014; Munck, 2006). Dadas las analogías entre las transformaciones sociales asociadas con la expansión de la economía de mercado en el pasado y el presente, se advierte que la tesis de Polanyi habla casi directamente a los problemas actuales (Stiglitz, 2003). Según sus planteamientos (Polanyi, 2003), el liberalismo del laissez-faire desarrollado por economistas políticos ingleses e implementado por varios Estados europeos en los siglos XIX y XX, requiere un desarraigo sistemático (dissembeding) de la economía respecto de la sociedad (que ignora el arraigo tradicional de la economía en las relaciones sociales). Este intento liberal de desarraigar la economía se lleva a cabo mediante la extensión de la lógica del mercado, a lo que él denomina las tres mercancías ficticias: la fuerza del trabajo, la tierra y el dinero. Una parte esencial de la crítica de Polanyi (2003) es que el desarraigar la economía de las relaciones sociales no es más que una utopía liberal, inalcanzable e insostenible. En particular, hay «una contradicción existencial entre los requerimientos de la economía de mercado capitalista para su expansión ilimitada y los de la gente para vivir en una relación de sociedad mutuamente comprensible» (Polanyi-Levitt, 2014:204). Tal contradicción es ejemplificada por la dinámica del doble movimiento que intenta proteger la sustancia humana y natural de la sociedad, así como su organización 
empresarial, en contra de su subordinación a la economía. Como lo documentó Polanyi (2003) en el escenario de la crisis económica, social y política del periodo de entreguerras europeo, este contramovimiento dio pie al desarrollo progresista de la protección social y fatalmente al fascismo retrógrado y a la guerra.

El auge del neoliberalismo representa asimismo un cambio fundamental en las instituciones y las relaciones económicas, políticas y sociales a través del mundo:

El proceso de neoliberalización ha acarreado un acusado proceso de «destrucción creativa» no sólo de los marcos y de los poderes institucionales previamente existentes (desafiando incluso las formas tradicionales de soberanía estatal) sino también de las divisiones del trabajo, de las relaciones sociales, de las áreas de protección social, de las combinaciones tecnológicas, de las formas de vida y de pensamiento, de las actividades de reproducción, de los vínculos con la tierra y de los hábitos del corazón (Harvey, 2007:7).

Los procesos de neoliberalización cobraron fuerza en la década de 1970 en medio de una crisis económica y política que afectó a los países capitalistas occidentales y que vio el fin del auge de la posguerra y el surgimiento de la estanflación. En el Norte global, la llegada al poder de Margaret Thatcher en Inglaterra y Ronald Reagan en Estados Unidos marcó el inicio de una revolución neoliberal definida por procesos de privatización, desregulación y comercialización (George, 1999). La difusión global del neoliberalismo puede atribuirse a factores como la crisis económica crónica de los 1980 y 1990 y el colapso del bloque comunista. Previamente se argumentó que las crisis mexicanas de 1982 y 1994 (Arias, 2015) y la crisis financiera asiática de 1997 (Kim, 2015) representan también coyunturas decisivas. El neoliberalismo se expandió globalmente después de tales crisis, cuando la lógica del mercado y procesos de privatización, desregulación y comercialización se institucionalizaron a través de programas de ajuste estructural y de austeridad fiscal.

Los impactos de esa transformación han variado entre y dentro de los países, aun así pueden apreciarse diversas tendencias relacionadas a la expansión de los mercados y a los procesos de privatización, desregulación y comercialización. En el caso de los países latinoamericanos, Alejandro Portes (1997b) identifica un número de tendencias comunes sintomáticas de esta transformación social neoliberal: a) la reevaluación de la ganancia capitalista como deseable y congruente 
con el interés nacional, b) la devaluación de los trabajadores sindicalizados y las industrias protegidas, c) la convicción de que la desigualdad social se puede enfrentar de modo más efectivo mediante el «efecto de goteo» de los mercados, y d) la reorientación de las fuentes de orgullo nacional hacia la inserción de la economía nacional en la economía mundial. En ese escenario de cambio continuo debe tomarse en cuenta el creciente papel de la «financiarización» en la economía contemporánea (que subraya el desplazamiento del poder y la captura de ganancias hacia las instituciones y las cadenas de valor globales financieras) (PolanyiLevitt, 2013).

Con base en la crítica polanyiana, la transformación social neoliberal — ejemplificada por la globalización — es un proceso diferenciado de inclusión y exclusión de determinadas regiones y grupos sociales en las relaciones de mercado capitalista mundial (Castles, 2007). Comprende no sólo el aumento de la desigualdad entre el Norte y el Sur globales, sino las desigualdades entre países y dentro de ellos. Por ende, es imprescindible situar la migración contemporánea frente a dichas desigualdades (Czaika y De Haas, 2014), porque, como lo destacó Zygmunt Bauman hace ya más de dos décadas, "la movilidad se ha convertido en el factor estratificador más poderoso y codiciado de todos; aquel a partir del cual se construyen y reconstruyen diariamente las nuevas jerarquías sociales, políticas, económicas y culturales de alcance mundial» (2001:16). Cabe aclarar que no se argumenta que una disminución de la desigualdad reduciría la migración de una manera causal. Este tipo de vinculación es peligroso, pues puede exacerbar una lógica de resolución de problemas que asume que ciertas formas de migración (en particular aquellas que han sido construidas como normativamente «malas» o "peligrosas») pueden y deben ser contenidas o reguladas. Ya se ha aludido que las migraciones no son fenómenos aislados ni anormales, de ahí que se insista en que el problema no es de causalidad sino de contexto. Es decir, si hubiera menos desigualdad en la era neoliberal (y, por ende, menos pobreza e inseguridad humana), no habría menos flujos migratorios, pero éstos ocurrirían en circunstancias muy diferentes a las actuales (Castles, 2010).

\section{La tesis polanyiana sobre la Gran Transformación}

Las ideas teóricas desarrolladas por Karl Polanyi (2003) pueden ser útiles para avanzar hacia una comprensión más matizada de la migración en el escenario de la 
expansión de la economía de mercado y los procesos asociados de transformación social. En primer lugar, el concepto de la economía arraigada evidencia que la noción de la economía de mercado, como un sistema independiente de mercados integrados capaces de autorregularse, es una ficción inalcanzable e insostenible. Polanyi (2003) destaca que nunca antes en la historia fueron los mercados más que accesorios de la vida social y que el sistema social nunca se separó de, ni fue subordinado, al económico. El concepto de la economía arraigada en sí reconoce la interconexión y la integridad de lo social, lo económico y lo político: ello significa que la economía nunca es verdaderamente autónoma, pues está siempre sujeta a formas de regulación política y a diversas normas morales, religiosas y éticas (Block, 2003). Dicho concepto también enfatiza un cambio ideológico y político radical asociado con el liberalismo del laissez-faire y el neoliberalismo.

En efecto, la expansión de la economía de mercado - que intenta romper los vínculos orgánicos y subordinar la sociedad y la naturaleza a la economía- es un proyecto profundamente político y no el resultado de ninguna evolución natural. De manera similar, y en concordancia con algunos críticos contemporáneos, la globalización neoliberal —en la cual nuevas formas de migración se han desarrollado - ha sido un proyecto político concebido en términos ideológicos (Petras y Veltmeyer, 2000). El énfasis dado por Polanyi (2003) al carácter arraigado de la economía en las relaciones sociales, y al carácter fundamentalmente político e ideológico de la transformación social, proporciona información importante sobre la naturaleza de la migración en la era neoliberal, en especial al hacer hincapié en el vínculo esencial entre los cambios en relaciones e instituciones económicas, sociales y políticas, que a menudo hace falta en otros análisis relativos a la migración contemporánea.

En segundo lugar, esta crítica de la economía de mercado se encuentra intrínsecamente ligada a la idea de las mercancías ficticias, misma que expone que la extensión de la lógica del mercado a los elementos sustanciales del trabajo, la tierra y el dinero es hondamente problemática e irracional. Frente a la lógica de que todos los elementos de la producción deben ser sujetos a las leyes de la oferta y la demanda, y que deben ser negociados en un mercado autorregulado, Polanyi (2003) subraya que incluir la fuerza trabajo, la tierra y el dinero bajo esa lógica subordina la sustancia real de la sociedad a las supuestas leyes del mercado. Se trata de un punto de contradicción: si bien el trabajo, la tierra y el dinero son esenciales para la economía de mercado, no son mercancías reales (en el sentido de que no son objetos producidos para su venta en el mercado) y 
su compraventa crea conflictos y peligros en curso. ${ }^{6}$ En opinión de Michael Burawoy (2015), la extensión de la organización del mercado a esas tres mercancías ficticias conduce a relaciones de inseguridad, despojo y endeudamiento que amenazan a comunidades, medios de vida y hábitats. Para los estudios de migración, la noción de la fuerza de trabajo como una mercancía ficticia, una que «no puede ser manipulada, usada indiscriminadamente, o incluso dejarse ociosa, sin afectar también al individuo humano que sea el poseedor de esta mercancía peculiar» (Polanyi, 2003:123), es la más destacada debido a la inseguridad que sufren muchos migrantes.

Por último, en tercer lugar, Polanyi (2003) expone que ninguna sociedad puede tolerar los efectos de la extensión de la organización del mercado, a menos que su sustancia humana y natural, al igual que su organización empresarial, estén protegidas. Un punto clave de su crítica de la economía de mercado autorregulado es que comprende una utopía inalcanzable e insostenible.? Afirma, además que inevitablemente la sociedad tomará medidas para defenderse a sí misma mediante un doble movimiento. En medio del auge del orden liberal y neoliberal, este contramovimiento constituye la interacción reactiva de dos principios de organización en la sociedad: el principio de liberalismo de laissez-faire y el principio de protección social. En concreto, mientras la economía de mercado se extiende, instrumentos, asociaciones y legislaciones progresistas y reaccionarias se crean con el propósito de supervisarla y regularla. Esta crítica sigue siendo relevante gracias al continuo crecimiento de los mercados en términos geográficos y reales, y dada la presencia de diversas luchas sociales y formas de resistencia contra ellos.

\footnotetext{
${ }^{6}$ Polanyi sostiene que la fuerza de trabajo «es sólo otro nombre para una actividad humana que va unida a la vida misma, la que a su vez no se produce para la venta sino por razones enteramente diferentes; ni puede separarse esa actividad del resto de la vida, almacenarse o movilizarse». Del mismo modo, la tierra «es otro nombre de la naturaleza, que no ha sido producida por el hombre», mientras que el dinero «es sólo un símbolo del poder de compra que por regla general no se produce sino que surge a través del mecanismo de la banca o de las finanzas estatales» (2003:123).

${ }^{7}$ En opinión de Polanyi, «tal institución no podría existir durante largo tiempo sin aniquilar la sustancia humana y natural de la sociedad; habría destruido físicamente al hombre y transformado su ambiente en un desierto» (2003:49).
} 


\section{Limitaciones del marco polanyiano para el estudio de la migración}

Aunque el trabajo de Karl Polanyi (2003) representa un modelo conceptual sólido para la crítica del neoliberalismo, es necesario abordar algunas de las limitaciones del marco original que restringen su poder analítico sobre formas contemporáneas de movilidad humana. Una inquietud es el dilema político planteado por el carácter ambivalente del doble movimiento en el marco teorético de Polanyi. Es decir, que las reacciones a la expansión de la economía de mercado pueden tomar una forma progresiva o una reaccionaria, esta última con frecuencia conduce a modos de opresión o xenofobia contra minorías como los migrantes (como se ha presenciado en muchos países del mundo en el pasado y en años más recientes) (Munck, 2006). Adicionalmente, a este dilema sobresalen dos temas analíticos interrelacionados que requieren ser abordados antes de poner en práctica las ideas de Polanyi en una investigación contemporánea: a) la necesidad de ir más allá del marco «nacional» en el análisis de los procesos de transformación social y de las formas vinculadas de acción humana y resistencia; y b) la necesidad de tomar en cuenta formas de desigualdad existentes que no pueden ser reducidas a, ni explicadas por, la expansión de la economía de mercado y los procesos asociados de privatización, desregulación y comercialización.

Primero, es preciso incluir otras escalas socioespaciales (junto con la «nacional») en el análisis de procesos de transformación social y formas conexas de acción humana y resistencia. Se puede argumentar que Polanyi estaba trabajando dentro de los parámetros de lo que ahora se llama el «nacionalismo metodológico» (Munck, 2006), en el que el escenario de crisis asociado con la expansión de la economía de mercado, la comunidad imaginada que se movilizaba como parte del doble movimiento, y los principales parámetros de la protección social, se plantearon principalmente en cuanto al Estado nación (Fraser, 2013). Sin embargo, dicho enfoque nacional en la actualidad es insuficiente en términos analíticos y en la arena real, en la que modos contemporáneos del doble movimiento pueden desarrollarse (Munck, 2004). Así pues, es útil adoptar una conceptualización más flexible de las maneras en que los procesos de transformación social —entre ellos la migración - son integrados y producidos en una gama de escalas mutuamente constituidas (Glick-Schiller y Caglar, 2009; Williamson, 2015; Xiang, 2013). Lo anterior no niega que el Estado nación siga siendo una unidad clave en términos 
económicos, sociales y políticos, pero enfatiza en la necesidad de explorar y problematizar los vínculos entre las dimensiones globales, nacionales, regionales y locales de la transformación social, y la forma en la que el doble movimiento se desarrolla a través de varios niveles de poder y en diferentes sitios socioespaciales. Tal perspectiva en particular resalta el vínculo primordial entre cambios masivos en las relaciones de poder político y económico global y procesos de transformación social subsiguientes (Castles, 2010). Ya que los cambios a escalas mundial y nacional son percibidos y experimentados en el nivel local, esta perspectiva permite conceptualizar cómo los migrantes han respondido, se han adaptado o han desafiado esa transformación. En efecto, se argumenta que ciertas formas de acción humana y resistencia, como estrategias de vida individuales o familiares (que pueden incluir la migración interna o internacional) o movimientos sociales, que exigen el respeto de los derechos humanos o la inclusión social de los migrantes y otras minorías, pueden ser conceptualizadas dentro del doble movimiento contemporáneo (Castles, 2015).

Segundo, es indispensable considerar formas de desigualdad que no pueden ser reducidas a, ni explicadas por, la expansión de la economía de mercado y los procesos asociados de privatización, desregulación y comercialización. Nancy Fraser explica que «enfocado en los daños que emanan de los mercados desarraigados, Polanyi pasa por alto los daños que se originan en otros lugares (...) y descuida las luchas contra injusticias enraizadas en la sociedad y codificadas en la protección social» (traducción del autor) (2011:140). El análisis de Polanyi se centra en el intento de subordinar la sociedad a la lógica del mercado (en particular en el nivel nacional), pero se mantiene taciturno acerca de la cuestión del colonialismo (Munck, 2015) y otras formas de desigualdad que tienen lugar en diversas escalas socioespaciales (Fraser, 2011). Al apelar a críticas feministas que ponen de manifiesto cuestiones de diferencia y situacionalidad (Lewis y Mills, 2003), se propone interrogar críticamente la manera en que jerarquías sociales, económicas y políticas, se complementan e interactúan entre sí con gran complejidad a través de múltiples escalas (Castles, 2005). Este replanteamiento problematiza el carácter ambivalente de la protección social tradicional, institucionalizada por los estados de bienestar y los Estados desarrollistas en la era de la posguerra, y que brindó alivio de los efectos negativos infligidos por la expansión de la economía de mercado en algunas comunidades, al tiempo que afianzó formas de desigualdad dentro y entre ellas (Fraser, 2013). Por ejemplo, varios aspectos de la protección social se sustentaron en la división entre el trabajo remunerado productivo y el trabajo no 
remunerado reproductivo en detrimento de las mujeres, o con base en normas culturales o religiosas de la mayoría en detrimento de las minorías étnicas o religiosas. De modo similar, el marco nacionalista de la protección social excluyó a muchos sujetos postcoloniales de sus beneficios - dentro y fuera del Estado nación-a pesar de los legados del colonialismo y su centralidad en la expansión de la economía de mercado. Las desigualdades, relacionadas con el estatus de las mujeres, de las minorías étnicas y religiosas, o de sujetos postcoloniales o racializados, son temas fundamentales para los debates contemporáneos sobre la migración (Castles, 2005; Parreñas, 2001; Sharma, 2008).

Tomando en cuenta esas limitaciones del marco polanyiano, es pertinente señalar que la comprensión de la migración en la era neoliberal puede fortalecerse mediante una mayor vinculación con la teoría social contemporánea que ha sido desarrollada en las últimas décadas con la finalidad de satisfacer un mundo cada vez más complejo, diverso e interconectado. Hacer una revisión completa de la teoría social relevante no es posible aquí, por lo que es preciso concentrarse en los enfoques teóricos utilizados en el proyecto de STIM. La discusión acerca de la transformación social como un marco de referencia se ha basado principalmente en las teorías de la globalización y el cambio social (Burawoy, 2000; Held et al., 1999; Munck, 2002; Stiglitz, 2002) y en análisis socioespaciales de la migración (Glick-Schiller y Caglar, 2009; Williamson, 2015; Xiang, 2013). También ha sido influenciada por desarrollos teóricos de estudios feministas, aquellos sobre la era y la sociedad de la información y los que abordan formas contemporáneas de resistencia y políticas de identidad, entre otros (Castles et al., 2011b; Fraser, 2013; 2014). En consecuencia, y aunque no es posible generar una síntesis integral en esta etapa, se reitera la importancia de integrar la investigación de la migración en un conocimiento más general y multidisciplinario de la sociedad. Complementariamente, se advierte que la elección de ejemplares de la teoría social refleja la propia capacitación disciplinaria de los investigadores miembros del equipo de investigación (en especial sociólogos, antropólogos, economistas políticos y estudiosos de las ciencias sociales), lo que enfatiza aún más la necesidad de una colaboración interdisciplinaria más amplia (Brettell y Hollifield, 2007). Finalmente, se reconoce la urgencia de incluir perspectivas y críticas del Sur global que puedan desafiar constructivamente las epistemologías dominantes y los propios etnocentrismos. 


\section{Los cimientos para una teoría de alcance intermedio}

El marco teórico presentado se diseñó para guiar y generar una discusión en torno de las maneras en que las formas de movilidad humana contemporánea interaccionan con instituciones y relaciones políticas, económicas y sociales a través de múltiples escalas socioespaciales en el escenario de relaciones cambiantes de poder e inequidad que caracterizan a la era neoliberal. Las proposiciones teóricas pueden considerarse una contribución al avance de un marco conceptual interdisciplinario de alcance intermedio en aras de analizar ciertas formas de migración que tienen lugar en el momento y contexto específicos de la era neoliberal (Castles, 2010; 2007). ${ }^{8}$ Con anterioridad, Stephen Castles (2015) ha identificado un número de principios clave que guían este marco teórico (y que ya se han discutido implícitamente), los cuales se describen a continuación:

1. La migración no es un resultado de la transformación social, ni una causa de la misma, sino una parte integral de dicho proceso. Se destaca la relevancia de conceptualizar la migración como un componente central de la transformación social neoliberal, arraigada en distintos cambios en las instituciones y relaciones políticas, económicas, sociales y culturales.

2. Es erróneo tratar de separar los estudios de migración de la teoría social más amplia. En una época de integración global, en la que las relaciones de poder e inequidad son cada vez más amplias y universales, la teoría social contemporánea no puede ignorar el crecimiento de la movilidad humana y los estudios de la migración tampoco pueden permanecer aislados de ella.

3. Los procesos migratorios son moldeados tanto por estructuras macrosociales como por las acciones y percepciones de las poblaciones afectadas en el nivel local. Por eso se propone un análisis crítico del papel de las estructuras transnacionales y nacionales, así como de la acción humana.

4. Las fuerzas globales son mediadas a través de patrones culturales y de experiencias históricas nacionales. Dado que las fuerzas globales que han impulsado la transformación social neoliberal son a menudo difíciles de percibir (porque los procesos asociados de cambio y de resistencia varían considerablemente de un Estado

${ }^{8}$ Robert Merton define una teoría de alcance intermedio como aquella que se encuentra «entre las hipótesis de trabajo menores, pero necesarias, que se producen abundantemente durante la investigación cotidiana y esfuerzos sistemáticos e integrales para desarrollar una teoría unificada que explique todas las uniformidades observadas del comportamiento, organización y cambio social» (traducción del autor) (1968:39). 
nación al otro), se sugiere que la investigación recurra a análisis transnacionales y a estudios nacionales para hacer sentido de dicha complejidad.

5. Los efectos de los cambios globales casi siempre se experimentan localmente en formas complejas. En tal escenario, la necesidad de emigrar o la llegada de nuevos grupos de inmigrantes con frecuencia sitúan a la migración y la diversidad como una de las caras más visibles del cambio en el nivel local. Al respecto, la investigación sobre la migración debe incluir las perspectivas y experiencias de los migrantes (y los no-migrantes) en esa escala.

Por lo tanto, entender el significado sociológico de la migración y de la transformación social en la era neoliberal requiere una investigación a través de múltiples escalas socioespaciales, que comprenda un análisis profundo de los vínculos y las contradicciones entre los niveles económicos, políticos y sociales. Así, la investigación acerca de la migración contemporánea tiene que ser multiescalar, multidisciplinaria e inclusiva del pensamiento y el conocimiento global.

Cabe aclarar que no se pretende que este «lente de transformación social» sea adecuado para explicar todas las formas de migración contemporánea, ni sus procesos asociados. Inclusive, se está de acuerdo con aquellos que cuestionan el valor y la viabilidad de una teoría general de la migración dada la complejidad y la diversidad del fenómeno en cuestión (Portes, 1997a; Portes y DeWind, 2004). Sin embargo, un marco de alcance intermedio centrado en el arraigo de la migración en los procesos de transformación social neoliberal puede revelar un entendimiento amplio de la migración. Según Alejandro Portes, en el contexto de teorías sociológicas de alcance intermedio, esto puede crear importantes narrativas de cambio, «incluyendo las múltiples contingencias y los retrocesos encontrados en el proceso (...) (así como) las restricciones estructurales y otros obstáculos que afectan específicamente a una búsqueda individual o colectiva» (traducción del autor) (1999:13). En ese sentido, esta propuesta teórica puede verse como los cimientos de dicha teoría de alcance intermedio. El contenido analítico de otras publicaciones basadas en la investigación primaria en Australia, Corea del Sur, México y Turquía demuestran algunos de los modos en que el marco puede ser puesto en práctica (Castles, Ozkul y Arias, 2015). Otros proyectos de investigación realizados por miembros del equipo de investigación (Arias, 2017; Kim, 2018) y por colegas en otras instituciones (De Haas y Fransen, 2018) también proporcionan ejemplos de investigación cualitativa y cuantitativa que utilizan ese marco para identificar y vincular formas contemporáneas de migración con dimensiones clave de la transformación social. Así, se espera que este marco teorético sea 
probado, criticado y desarrollado por otros investigadores con el objetivo común de adquirir una mejor comprensión de las profundas interconexiones entre la movilidad humana y los procesos multiescalares de transformación política, económica y social en la era neoliberal.

\section{Referencias}

Alatas, Syed (2003), "Academic dependency and the global division of labour in the Social Sciences», Current Sociology, 51(6), pp. 599-613.

Albrow, Martin (1996), The golden age, Cambridge, Polity.

Arango, Joaquin (2004), «Theories of international migration», en Danièle Joly, International migration in the new millennium, Burlington, Ashgate, pp. 15-35.

Arias Cubas, Magdalena (2015), «From Casa Blanca to Tulsa: a social transformation analysis of Mexican migration in an era of neoliberal globalization", en Stephen Castles, Derya Ozkul y Magdalena Arias Cubas, Social transformation and migration: national and local experiences in South Korea, Turkey, Mexico and Australia, Basingstoke, Palgrave Macmillan, pp. 169-184. (2017), International migration and social transformation in the neoliberal era: a case study of mixtecos as indigenous people in Mexico and irregular migrants in the US (tesis de doctorado), Sydney, University of Sydney.

Bauman, Zygmunt (2001), La globalización. Consecuencias humanas (traducido por Daniel Zadunaisky), México, Fondo de Cultura Económica.

Beck, Ulrich (2007), «Beyond class and nation: reframing social inequalities in a globalizing world», The British Journal of Sociology, 58(4), pp. 679-705.

Block, Fred (2003), «Introducción», en Karl Polanyi, La gran transformación: los orígenes políticos y económicos de nuestro tiempo, México, Fondo de Cultura Económica, pp. 21-41.

Brettell, Caroline y James Hollifield (2007), Migration theory: talking across disciplines, London, Routledge.

Burawoy, Michael (2000), "A sociology for the second great transformation?», Annual Review of Sociology, 26, pp. 693-695.

Burawoy, Michael (2015), "Facing an unequal world», Current Sociology, 63(1), pp. 5-34.

Castells, Manuel (1996), The rise of the network society, Oxford, Blackwell. (1997), The power of identity, Oxford, Blackwell. (1998), End of millenium, Oxford, Blackwell. 
Castles, Stephen (2004), "Why migration policies fail?», Journal of Ethnic and Migration Studies, 27(2), pp. 205-227.

(2005), «Nation and empire: hierarchies of citizenship in the new global order», International Politics, 42, pp. 203-224.

(2007), «Twenty-first-century migration as a challenge to sociology», Journal of Ethnic and Migration Studies, 33(3), pp. 351-371.

(2010), «Understanding global migration: a social transformation pers-

pective», Journal of Ethnic and Migration Studies, 36(10), pp. 1565-1586.

(2015), «International human mobility: key issues and challenges to so-

cial theory», en Stephen Castles, Derya Ozkul y Magdalena Arias Cubas, Social transformation and migration: national and local experiences in South Korea, Turkey, Mexico and Australia, Basingstoke, Palgrave Macmillan, pp. 3-14.

Castles, Stephen, Magdalena Arias Cubas, Chulhyo Kim, Elsa Koleth, Derya Ozkul, y Rebecca Williamson (2011a), «Karl Polanyi's great transformation as a framework for understanding neo-liberal globalisation", Social transformation and international migration project: working paper 1, Sydney, University of Sydney.

(2011b), "Rethinking social transformation theory in the 21st century», Social transformation and international migration project: working paper 2, Sydney, University of Sydney.

Castles, Stephen y Raúl Delgado Wise (2008), «Introduction», en Stephen Castles y Raúl Delgado Wise, Migration and development: perspectives from the South, Geneva, International Organisation of Migration, pp. 1-13.

Castles, Stephen, Derya Ozkul y Magdalena Arias Cubas (2015), Social transformation and migration: national and local experiences in South Korea, Turkey, Mexico and Australia, Basingstoke, Palgrave Macmillan.

Connell, Raewyn (2007), Southern theory: the global dynamics of knowledge in Social Science, Crows Nest, Allen \& Unwin.

Connell, Raewyn (1997), "Why is classical theory classical?», American Journal of Sociology, 102(6), pp. 1511-1557.

Czaika, Mathias y Hein de Haas (2014), "The globalization of migration: has the world become more migratory?.», International Migration Review, 48(2), pp. 283-323.

De Haas, Hein (2014), "Migration theory-quo vadis?», IMI Working Paper 100, Oxford, International Migration Institute.

De Haas, Hein y Sonja Fransen (2018), «Social transformation and migration: an empirical inquiry», en https://www.imi-n.org/publications/social-transformation -and-migration-an-empirical-inquiry 
De Sousa Santos, Boaventura, Joao Arriscado Nunes y Maria Meneses (2007), «Introduction: opening up the canon of knowledge and recognition of difference», en Boaventura de Sousa Santos, Another knowledge is possible: beyond northern epistemologies, London, Verso, pp. XIX-LXII.

Faist, Thomas (2000), The volume and dynamics of international migration and transnational social spaces, Oxford, Oxford University Press.

Fraser, Nancy (2011), «Marketization, social protection, emancipation: toward a neopolanyian conception of capitalist crisis», en Craig Calboun y Georgi Derluguian, Business as usual: the roots of the global financial meltdown, New York, NYU Press, pp. 137-157.

Fraser, Nancy (2013), «A triple movement? Parsing the politics of crisis after Polanyi», New Left Review, 81, pp. 119-32.

Fraser, Nancy (2014), "Can society be commodities all the way down? Post-polanyian reflections on capitalist crisis», Economy and Society, 43(4), pp. 541-558.

George, Susan (2012), "A short history of neoliberalism», en http://www.tni.org/ article/short-history-neoliberalism

Glick-Schiller, Nina y Ayse Caglar (2009), "Towards a comparative theory of locality in migration studies: migrant incorporation and city scale», Journal of Ethnic and Migration Studies, 35(2), pp. 177-202.

Harvey, David (2007), Breve historia del neoliberalismo (traducido por Ana Varela Mateos), Madrid, Ediciones Akal.

Held, David, Anthony McGrew, David Goldblatt y Jonathan Perraton (1999), Global transformations: politics, economics, and culture, Cambridge, Polity.

Kim, Chulhyo (2015), «From salt fields to «multicultural village special zones: the transformation of a migrant city in South Korea», en Stephen Castles, Derya Ozkul y Magdalena Arias Cubas, Social transformation and migration: national and local experiences in South Korea, Turkey, Mexico and Australia, Basingstoke, Palgrave Macmillan, pp. 97-112.

Kim, Chulhyo (2018), Temporary labour migration, social movements and neoliberal transformation in South Korea (tesis de doctorado), Sydney, University of Sydney.

King, Russell (2012), "Theories and typologies of migration: an overview and a primer», en Willy Brandt Series of Working Papers in International Migration and Ethic Relations 3/12, Malmö, Malmö Institute for Studies of Migration, Diversity and Welfare.

Lee, Jennifer, Jørgen Carling y Pia Orrenius (2014), «The international migration review at 50: reflecting on half a century of international migration research and looking ahead», International Migration Review, 48(1), pp. 3-36. 
Levitt, Peggy (2004), "Transnational migrants: when home» means more than one country", en http://www.migrationinformation.org/feature/display.cfm? $\mathrm{ID}=261$

Lewis, Reina y Sara Mills (2003), «Introduction», en Reina Lewis y Sara Mills, Feminist postcolonial theory: a reader, Edinburgh, Edinburgh University Press, pp. 1-21.

Massey, Douglas, Joaquin Arango, Graeme Hugo, Ali Kouaouci, Adela Pellegrino y J. Edward Taylor (1998), Worlds in motion: understanding international migration at the end of the millenium, New York, Oxford University Press.

Merton, Robert (1968), Social theory and social structure, New York, Free Press.

Munck, Ronaldo (2002), "Globalisation and democracy: a new sgreat transformation?.», The Annals of the American Academy of Political and Social Science, 581, pp. 10-21.

(2004), «Globalisation, labour and the «Polanyi Problem», Labour History, 45(3), pp. 251-269.

(2006), «Globalization and contestation: a polanyian problematic», Globalizations, 3(2), pp. 175-186.

(2015), «Globalisation, trade unions, and labour migration: a transformationalist approach", en Carl Schierup, Ronaldo Munck, Branka Likic-Brboric y Anders Neergaard, Migration, precarity and global governance: challenges and opportunities for labour, Oxford, Oxford University Press, pp. 101-117.

Park, Robert (1950), Race and culture, Glencoe, The Free Press.

Parreñas, Rhacel (2001), Servants of globalization: women, migration and domestic work, Standford, Stanford University Press.

Petras, James y Henry Veltmeyer (2000), «Globalisation or imperialism?», Cambridge Review of International Affairs, 14(1), pp. 32-48.

Polanyi-Levitt, Kari (2013), From the great transformation to the great financialization: on Karl Polanyi and other essays, New York, Zed Books.

(2014), «Los conceptos más importantes en el trabajo de Karl Polanyi y su relevancia contemporánea», Economía y Desarrollo, 151, pp. 198-211.

Polanyi, Karl (2003), La gran transformación: los orígenes políticos y económicos de nuestro tiempo, México, Fondo de Cultura Económica.

Portes, Alejandro (1997a), «Immigration theory for a new century: some problems and opportunities», International Migration Review, 31(4), pp. 799-825.

(1997b), «Neoliberalism and the sociology of development: emerging trends and unanticipated facts», Population and Development Review, 23(2), pp. $229-259$. 
(1999), "The hidden abode: sociology as analysis of the unexpected", American Sociological Review, 65(1), pp. 1-18.

Portes, Alejandro y Josh DeWind (2004), "A cross-Atlantic dialogue: the progress of research and theory in the study of international migration", International Migration Review, 38(3), pp. 828-851.

Rosewarne, Stuart (2010), "Globalisation and commodification of labour», The Economic and Labour Relations Review, 20(2), pp. 99-110.

Sharma, Nandita (2008), "Citizenship and the disciplining of (im)migrant workers in the United States», en Sonia McKay, Refugees, recent migrants and employment: challenging barriers and exploring pathways, London, Routledge, pp. 102-120.

Skeldon, Ronald (1997), Migration and development: a global perspective, Essex, Longman.

Stiglitz, Joseph (2002), Globalization and its discontents, London, Penguin. (2003), «Prólogo», en Karl Polanyi, La gran transformación: los orígenes politicos y económicos de nuestro tiempo, México, Fondo de Cultura Económica, pp. 9-19.

Tuhiwai Smith, Linda (1999), Decolonizing methodologies: research and indigenous peoples, London, Zed books.

Williamson, Rebecca (2015), «Towards a multi-scalar methodology: the challenges of studying social transformation and international migration», en Stephen Castles, Derya Ozkul y Magdalena Arias Cubas, Social transformation and migration: national and local experiences in South Korea, Turkey and Australia, Basingstoke, Palgrave, pp. 17-32.

Wimmer, Andreas y Nina Glick Schiller (2003), "Methodological nationalism, the social sciences, and the study of migration: an essay in historical epistemology», International Migration Review, 37(3), pp. 576-610.

Xiang, Biao (2013), «Multi-scalar ethnography: an approach for critical engagement with migration and social change», Ethnography, 14(3), pp. 282-299. 